but also contain guidance on the use of levers, crankcases and wheels that are fitted to work stations.

Methods This paper presents the results of several research studies performed by NIOM researchers in the field of force necessary for the professional activities. The studies were done using the measuring set: tensometric dynamometer, force converter, amplifier and PC for data collection. Measurements were done at least 3 times for one activity, the average value of force was taken for further analysis.

Result During the tests, it was stated that, depending on the technical condition of the transport trolleys used in the hypermarkets (weight including the load of about $450 \mathrm{~kg}$ limited by law), kind of the pavement on which they moved and the way they were put into motion, the force necessary to start their movement was from $60 \mathrm{n}$ to $650 \mathrm{n}$.

Discussion These values were 2-3 times higher than the applicable standards (300 n - for pushing, $250 \mathrm{n}$ - for pulling). On the other hand, the measurements of the force needed to launch overloaded trucks used in the transport of gas cylinders or materials in the textile industry (up to about $1500 \mathrm{~kg}$ ) indicate the necessity to use $500 \mathrm{n}-700 \mathrm{n}$ force - well above the permissible. It is visible that the ergonomic interventions including education and training is necessary.

\section{BIOMECHANICAL AND PHYSIOLOGICAL PARAMETERS FOR MANUAL MATERIAL HANDLING (MMH) RISK ASSESSMENT IN MARITIME WORKERS}

${ }^{1} \mathrm{~A}$ Silvetti*, ${ }^{2} \mathrm{E}$ Munafò, ${ }^{1} \mathrm{~A}$ Ranavolo, ${ }^{1} \mathrm{~F}$ Draicchio. ${ }^{1}$ INAIL, Monte Porzio Catone, Italy; 'ITAL UIL, Rome, Italy

\subsection{6/oemed-2018-ICOHabstracts.769}

Introduction According to EU data, the fishing sector is the one with the highest injury rate of all other sectors. Aim of the study is to assess some $\mathrm{MMH}$ tasks in which it was possible to use the common standardised protocols in conditions where, although their restrictions, they could provide early indications.

Methods NIOSH protocol was used to assess unloading crates of fish (weight of $12 \mathrm{Kg}$ or $16 \mathrm{Kg}$ ) out of the boat to the van and while unloading crates, inside the boat, from the refrigerator to the slipway. Unloading crates from the boat to the van was studied also by means of 3DSSPP to estimate compression force at L4/L5 level. Heart rate monitors were used to estimate CCr while handling crates inside refrigerator. Result Results obtained using the NIOSH protocol show LIs between 2.55 and 6.34 and a RWL between $2.52 \mathrm{Kg}$ e $4.69 \mathrm{Kg}$. 3DSSPP analysis reported L4/L5 compression force ranging between $2752 \mathrm{~N}$ and $3946 \mathrm{~N}$ and low strength percent capability at wrist, shoulder, trunk and hip joints. Unloading crates from refrigerator to slipway analysis reported LIs ranging from 1.63 to 5.83 and a RWL ranging from $2.74 \mathrm{Kg}$ to $7.36 \mathrm{Kg}$. $\mathrm{MMH}$ inside refrigerator showed CCr values of $40.5 \%$ and $42.7 \%$.

Discussion During boarding, we observed several activities worthy of attention under the biomechanical overload point of view. We investigated the most strenuous tasks according to the crew's information. All obtained values were largely over the limit for all used methods and are consistent with results from other studies. We observed MMH activities not assessable with any of the methods currently available in the literature because they cannot describe the tasks in their globality. It was also noted that the workers, based on their experience, have adopted enhancements reducing vertical displacement, asymmetry angle and adopting an internal organisation of rotation during $\mathrm{MMH}$ tasks.

Acknowledgements This study has been accomplished with the support from UILA Pesc

\section{DOCKWORKERS MUSCULOSKELETAL INJURY PREVENTION PROGRAM ON A BRAZILIAN TERMINAL}

${ }^{1} \mathrm{~EB}$ Lima*, ${ }^{2} \mathrm{MM}$ Almeida, ${ }^{3} \mathrm{AS}$ Alves, ${ }^{4} \mathrm{MA}$ Pinto, ${ }^{5} \mathrm{FF}$ Santos. ${ }^{1}$ Occupational Physician in Terminal Exportador do Guarujá (TEG); ${ }^{2}$ In charge of Quality of Life (TEG); ${ }^{3}$ Work Nurse (TEG); ${ }^{4}$ QMSS Supenvisor (TEG); ${ }^{5}$ Operation Manager (TEG)

\subsection{6/oemed-2018-ICOHabstracts.770}

Introduction Terminals in the port of Santos, the largest in Latin America, are constantly working to minimise physical impacts of work on employees. Aiming at the prevention of musculoskeletal injuries and quality of life improvement, we implemented a series of functional and ergonomic measures in 2014, which resulted in significant absenteeism reduction and excellent acceptance from employees, with a relevant improvement to their quality of life.

Methods The program begins with a health evaluation on the employee's admission that includes anthropometry, dynamometry and surface electromyography. On the first month of work, the employee's last $40 \mathrm{~min}$ of the workday are held in a gym inside the terminal, where specific exercises, conducted by 3 physical educators professionals. After this period this routine becomes optional, but with a strong incentive for its continuity (outside of the work period). Also, there is a daily labour gymnastics program maintained for all employees. Concomitantly, research is done on ergonomic conditions, where employees give opinions about job satisfaction and possible adverse conditions, suggesting actions that will be evaluated by the Ergonomics Committee that study and treat these cases. Result Since 2015, when the program started, if compared to 2014 , there was a $30 \%$ reduction of absenteism caused by osteomuscular injuries. From 2015 to 2016 there was a 46\% absenteism reduction from the same causes. When comparing 2014 with 2016 , there was an overall reduction of $62 \%$. Additionally, in 2016, 132 employees suggestions on ergonomic aspects were raised and addressed by the Ergonomics Committee. A relevant index show that $78 \%$ of the employees were practicing some physical activity by the end of 2016 and of those, $48 \%$ performed the activities inside the Terminal's gym.

Discussion Musculoskeletal injuries are the major causes of dockworkers absenteeism. The Program implementation and employees suggestions significantly reduced absenteeism reates, encouraged the physical activities practice in the workplace and consequently improved their quality of life.

\section{A PRACTICAL NOVEL MODEL FOR OFFICE ERGONOMICS AWARENESS AMONGST GLOBAL CORPORATE OFFICE EMPLOYEES'. SHARING EXPERIENCE FROM 11 COUNTRIES}

Kishore Madhwani. Hindustan Unilever Limited, Mumbai, India

\subsection{6/oemed-2018-ICOHabstracts.771}

Introduction With increase in use of laptops, globally there is a need to spread awareness on office ergonomics. The 
challenge lies in educating maximum employees in shortest time thereby reducing musculoskeletal ailments and promoting safer working by exploring effective communication methods.

Methodolgy A cross-sectional study encompassing Unilever Global Corporate Office Employees' is being undertaken [2013-2017] in India, Dubai(U.A.E), Nairobi (Africa), Durban (South Africa) and South East Asian countries [Philippines, Vietnam, Indonesia, Singapore, Malaysia, Thailand and Sri Lanka] $\mathrm{n}=$ Total 2646 .

Employees were grouped into two, to study following interventions;

- Lecture training including a forty-minute power point presentation \& demonstration on a mock office workstation educating employees on maintaining ideal work postures, back $\&$ eye protection, taking rest breaks, performing desk stretches $\&$ arranging workstations ergonomically to their body dimensions'. $\mathrm{n}=1546$.

- Short demonstrations (ten minutes) on each office floor on a live workstation educating employees on same parameters'. $\mathrm{n}=1100$.

Results Forty minute Lecture training enhances awareness in $>92 \%$ employees'. However, few employees attend this training, due to hectic work schedules.

Short ten minute live demonstrations undertaken in above countries appears to be an excellent tool enhancing awareness in $>90 \%$ employees. When both above interventions were tested for statistical significance, $40 \mathrm{~min}$ lecture was superior only in Indonesia $(\mathrm{p}<0.05)$.

Discussion As few employees attend $40 \mathrm{~min}$ lecture training (though superior) on office ergonomics, the ten minute live demonstration is a promising practical novel intervention as it is comprehensive, undertaken at the workstation, enhancing awareness in maximum employees in a short-time, instilling a feeling of caring and bonding which is vital for a successful and robust office ergonomics control program. A reminder card with tips on chair adjustments, ergonomic arrangement of workstations and a link on desk stretches serves as handy desk-reminder emphasising safer work postures.

\section{REPEATED BACK PAIN AND ROUTES OF EXIT OUT OF PAID EMPLOYMENT AMONG BRITISH CIVIL SERVANTS: A FOLLOW-UP STUDY 1985-2013}

\begin{abstract}
${ }^{1,2} \mathrm{~T}$ Lallukka*, ${ }^{2} \mathrm{M}$ Mänty, ${ }^{3} \mathrm{C}$ Cooper, ${ }^{4} \mathrm{M}$ Fleischmann, ${ }^{5,6,7} \mathrm{~A}$ Kouvonen, ${ }^{4} \mathrm{~J}$ Head, ${ }^{1} \mathrm{JI}$ Halonen. ${ }^{1}$ Finnish Institute of Occupational Health, Helsinki, Finland; ${ }^{2}$ Department of Public Health, University of Helsinki, Helsinki, Finland; ${ }^{3} M R C$ Lifecourse Epidemiology Unit, Universities of Southampton and Oxford, UK; ${ }^{4}$ Institute Department of Epidemiology and Public Health, University College London, London, UK; ${ }^{5}$ Department of Social Research, University of Helsinki, Helsinki, Finland; ${ }^{6}$ SWPS University of Social Sciences and Humanities in Wroclaw, Wroclaw, Poland; ${ }^{7}$ Administrative Data Research Centre - Northern Ireland, Queen's University Belfast, Belfast, UK
\end{abstract}

\subsection{6/oemed-2018-ICOHabstracts.772}

Introduction Pain is a risk factor for work disability, however, routes of exit out of paid employment among those with chronic pain have not been examined in detail. We aimed to examine the contribution of chronic back pain to subsequent transitions out of paid employment, accounting for covariates. Methods We included participants of the Whitehall II study cohort $(n=8445,69 \%$ men, aged $35-55$ at baseline), with measurements of back pain between phases 1 and 3 (19851994). Exit from paid employment (health-related, retirement not related to health, unemployment, other) was observed between 1995-2013 (phases 4-11). Those remaining in paid employment served as the reference group. Sex, age, parental and own socioeconomic position, job demands, job control, and body mass index were controlled for. Repeated measures logistic regression models were fitted.

Result Altogether 10\% of the participants exited paid employment due to health-related reasons, $2 \%$ due to unemployment and further $6.5 \%$ due to other reasons. After full adjustments, reporting back pain at one time point (26\%) was unassociated with exit due to health reasons, whereas reporting repeated pain $(18 \%)$ was associated with such exit (OR 1.53, 95\% CI: 1.17 to 2.00), when compared to those who did not report pain during phases 1-3 (56\%). Associations were somewhat stronger among middle or lower class employees, and nonexistent among high class employees. Otherwise differences e. g. by age, working conditions or obesity were small. The risk of exit due to other routes than health-related did not vary between participants with or without pain.

Discussion These results highlight the need for early detection of chronic pain to prevent the risk of health-related early exit out of paid employment. The results further emphasise the importance of identification of high risk groups and their modifiable risk factors, such as adverse working conditions

\section{THE TRENDS AND DETERMINANTS OF WORK-RELATED MUSCULOSKELETAL DISORDERS (MSD) IN IRELAND BETWEEN 2002 AND 2013}

Helen Russell*, Bertrand Maitre, Oona Kenny, Dorothy Watson. Economic and Social Research Institute, Dublin, Ireland

\subsection{6/oemed-2018-ICOHabstracts.773}

Introduction In Ireland between 2002-2013, Musculoskeletal Disorders (MSD) accounted for 50\% of self-reported workrelated illnesses. Moreover the average number of days absent (15.9 days) was higher than the average of 12.8 days for all other illnesses (except stress, anxiety and depression).

Methods This paper examines trends and determinants for work-related MSD between 2002 and 2013, using annual cross-sectional data from the Quarterly National Household Survey (QNHS).

Results Rates of MSD were strongly linked to the economic cycle. Rates per 1000 workers ranged from 11 in 2002 to 19 during the economic boom before falling to 7 during the recession (2009). The 2013 rate in a recovering economy was 14 per 1000 workers.

This pro-cyclical pattern remained when characteristics of workers and their workplace were held constant using logistic regression. Furthermore, within sectors, rates were higher when the annual percentage change in employment was positive.

We also found that certain worker and workplace factors influenced the risk of MSD independently. Workers aged 3564 had the highest risk of MSD (2.5 times more than workers $<25$ years). Construction sector workers, followed by those working in agriculture and health, had the greatest risk of MSD. Rates in education and all other services sectors were much lower. The self-employed, those working 40 to 49 hours per week (compared to $<30$ hours), shift workers, and new recruits (with $<6$ months job experience) also had a higher risk of MSD. 